\title{
Reproducibility in the Open Access Era for Biomedical Research
}

\section{Shu-Zong Wang ${ }^{1^{*}}$ and Eun Ji Gang ${ }^{2}$}

${ }^{1}$ Howard Hughes Medical Institute \& Department of Molecular, Cell and Cancer Biology, University of Massachusetts Medical School, Worcester, MA, USA ${ }^{2}$ Division of Hematology and Oncology, Children's Hospital Los Angeles, University of Southern California Keck School of Medicine, Los Angeles, CA, USA

"Corresponding author: Shu-Zong Wang, Howard Hughes Medical Institute, Department of Molecular, Cell and Cancer Biology, University of Massachusetts Medical School, Worcester, MA, USA 01605, Tel: 508-856-5337; E-mail: Phillip.Wang@umassmed.edu

Received date: April 07, 2016; Accepted date: April 11, 2016; Published date: April 13, 2016

Copyright: ( 2016 Wang SZ, et al. This is an open-access article distributed under the terms of the Creative Commons Attribution License, which permits unrestricted use, distribution, and reproduction in any medium, provided the original author and source are credited.

\section{Editorial}

In the past three decades, we have witnessed the tremendous expansion of scientific research, particularly in the biomedical field, as millions of researchers involved, billions of dollars invested --- for example, NIH is the main funder of academic biomedical research in US, distributing nearly $\$ 31$ billion per year in research grants, and large amount of papers published --- nearly 1,000,000 biomedical articles published every year. Today, the expansion has been even thought to be overdone and has created an unstainable hypercompetitive system with systemic flaws $[1,2]$.

Given the growth of the biomedical research, now it appears the time to translate these preclinical discoveries into drug invention to treat patients and cure diseases. In academia, this effort was highlighted by the establishment of a shiny NIH center, The National Center for Advancing Translational Sciences (NCATS), launched in January 2012, aiming to expedite to get new drugs, devices and diagnostics during drug development [3]. In the industry of biotechnology, the investment in drug development is predicted to keep increasing annually at a compound growth rate of $2.4 \%$ from 2013 to 2020, bringing the total annual funding to a staggering 165 billion dollars worldwide [4].

It appears that it is the time to pick up the ripe fruits. Nevertheless, some red flags have been recently thrown into the field. In 2011 and 2012, two giant pharma/biotech companies Amgen (California) and Bayer (Germany) published their findings in Nature, respectively, showing that only $10 \%$ to $30 \%$ of published scientific studies were actually reproducible $[5,6]$. This had become a shocking blockbuster in the biomedical research community as well as in the general public as the findings were further publicized in the Wall Street Journal and other general media. People would think them to be impossible and unbelievable and even to sabotage the integrity and reliability of biomedical research and scientists when they read about these numbers, because without reproducibility, science would not be science. Furthermore, the non-reproducibility has many more negative impacts. For example, some non-reproducible preclinical publications had generated even a new field and it was followed by hundreds of secondary publications that were based on the primary nonreproducible results [6]. Obviously, this has wasted tremendous amount of taxpayers' money, private funds, and other research resources.

As results, recently, people have taken actions to fight against it and attempted to tackle the problem. In October 2013, The Reproducibility Project-Cancer Biology was initiated by the collaboration between Science Exchange and the Center for Open Science. The former, Science Exchange, was founded by breast cancer biologist Elizabeth Iorns, and the latter, Charlottesville, Virginia-based COS, was founded with the $\$ 5.25$ million financial support by The Arnold Foundation, a Houston, Texas based foundation with a history of supporting efforts for the openness, integrity and reproducibility of biomedical research. The project, awarded \$1.3 million from the Arnold Foundation in 2013, aimed to replicate about 50 high-profile "landmark" papers published in top journals including Nature, Science, PNAS during 2010-2012. The replication results would be published in a Replication Study in eLife. Also, in the field of psychological research, a field closer to neurology and neurophysiology, a similar project---Reproducibility Project, was initiated to replicate published studies, which was also funded by the Center for Open Science [7-9].

However, this endeavor just gets started and is far from over since building a strong system remains a huge challenge. I would call the whole process towards a more rigorous system a revolution regarding the overall scale and the massive difficulty since the problem has "plagued scientific research for years" as mentioned by William Gunn, co-director of the Reproducibility Project. The reproducibility issue has been long and almost everywhere, therefore, it involves the change of the research culture in the whole community and it affects the interest of the majority. Definitely it is a long march but a deserving one for great efforts.

JNN is an open access journal and can definitely play some active roles in this endeavor. Its expedition and conciseness style can help publish those reproducible and robust results quickly which otherwise could not be readily published in other high profile journals which normally require many more data and experiments during a lengthy publishing cycle. Furthermore, negative results should be encouraged here, particularly the ones contradictory to those published in high profile papers or journals such as Nature, Science and Cell regarding to their key finding points. Reproducibility has become so critical an issue both practically and theoretically at present time for the future drug development and the long-term sustainable and healthy biomedical advancement that the highlighting it in the preclinical study phase could not be overemphasized.

\section{References}

1. Alberts B, Kirschner MW, Tilghman S, Varmus H (2014) Rescuing US biomedical research from its systemic flaws. Proc Natl Acad Sci U S A 111: 5773-5777.

2. Garrison HH, Justement LB, Gerbi SA (2016) Biomedical science postdocs: an end to the era of expansion. FASEB J 30: 41-44.

3. Wadman M (2012) NIH director grilled over translational research center, Nature News.

4. Lo A (2015) Can megafunds boost drug research? World Economy Forum 2015 December 
Citation: Wang SZ, Gang EJ (2016) Reproducibility in the Open Access Era for Biomedical Research. J Neurol Neurophysiol 7: e117. doi: 10.4172/2155-9562.1000e117

Page 2 of 2

5. Prinz F, Schlange T, Asadullah K (2011) Believe it or not: how much can we rely on published data on potential drug targets?. Nature Rev Drug Discov 10: 712 .

6. Begley CG, Ellis LM (2012) Drug development: Raise standards for preclinical cancer research. Nature 483: 531-533.

7. Van Noorden R (2013) Initiative gets $\$ 1.3$ million to verify findings of 50 high-profile cancer papers. Nature News.
8. Couzin-Frankel J (2013) Complete. Repeat? Initiative Gets \$1.3 Million to Try to Replicate Cancer Studies, Science News.

9. Wadman M (2013) NIH mulls rules for validating key results. Nature 500: 14-16. 